\title{
Correction to: Inhibitor of growth 2 regulates the high glucose-induced cell cycle arrest and epithelial-to-mesenchymal transition in renal proximal tubular cells
}

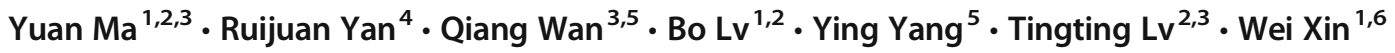 \\ Published online: 19 June 2020 \\ (C) University of Navarra 2020
}

\section{Correction to: Journal of Physiology and Biochemistry} https://doi.org/10.1007/s13105-020-00743-3

The above article was published online first with the following errors which have now been corrected.

Co-first authors' Correction: Yuan Ma, Ruijuan Yan, and Qiang Wan contributed equally to this work.

We apologize to the journal and readers for the error. We appreciate the opportunity to correct the data in our study.

Publisher's note Springer Nature remains neutral with regard to jurisdictional claims in published maps and institutional affiliations.

The online version of the original article can be found at https://doi.org/ 10.1007/s13105-020-00743-3

Wei Xin

weixin@mail.sdu.edu.cn

1 Department of Central Laboratory, Shandong Provincial Hospital, Cheeloo College of Medicine, Shandong University, Jinan 250021, Shandong, China

2 School of Medicine, Cheeloo College of Medicine, Shandong University, Jinan 250012, Shandong, China

3 Department of Endocrinology, Shandong Provincial Hospital, Cheeloo College of Medicine, Shandong University, Jinan 250021, Shandong, China
4 Department of Emergency Medicine, Shandong Provincial Third Hospital, Cheeloo College of Medicine, Shandong University, Jinan 250000, Shandong, China

5 Department of Endocrinology, Shandong Provincial Hospital, Affiliated to Shandong First Medical University, Jinan 250021, Shandong, China

6 Department of Central Laboratory, Shandong Provincial Hospital, Affiliated to Shandong First Medical University, Jinan 250021, Shandong, China 